'Integrantes del Grupo de Estudios: Carlos Echeverría B. (Presidente del Grupo de Estudios; Hospital Naval "Almirante Nef"), Alejandro Goic G. (Academia de Medicina del Instituto de Chile), Carolina Herrera C. (Clínica Avansalud), Carlos Quintana V. (Facultad de Medicina, Pontificia Universidad Católica de Chile), Alberto Rojas O. (Facultad de Medicina, Universidad Andrés Bello), Gonzalo Ruiz-Esquide (Clínica Santa

María), Rodrigo Salinas R. (Facultad de Medicina, Universidad de Chile),

Alejandro Serani M. (Facultad de Medicina, Universidad de los Andes), Paulina Taboada R. (Centro de Bioética, Pontificia Universidad Católica de Chile), Ricardo Vacarezza Y. (Facultad de Medicina, Universidad de Chile). Santiago, Chile.

Texto aprobado como Documento Oficial de la Sociedad Médica de Santiago-Sociedad Chilena de Medicina Interna.

Recibido el 20 de enero de 2015, aceptado el 26 de enero de 2015.

Este trabajo no cuenta con grants ni financiamiento externo. Los autores declaran no tener conflictos de intereses. La Dra. Carolina Herrera C. informa que tiene un contrato de trabajo con el Laboratorio Novartis, Chile, donde se desempeña como Clinical Advisor Respiratory.

Correspondencia a: Dr. Carlos Echeverría B. Hospital Naval "Almirante Nef". Subida Alessandri $s / n$. Viña del Mar. Chile.

Teléfono: +5632 2573380 ciecheverria@hotmail.com

\section{Algunas amenazas actuales a la confidencialidad en medicina}

\author{
GRUPO DE ESTUDIOS DE ÉTICA CLÍNICA DE LA \\ SOCIEDAD MÉDICA DE SANTIAGO ${ }^{1}$
}

\section{Some current threats to confidentiality in medicine}

Social, technical and legal conditions of the current practice of medicine make it necessary to insist on certain actions and circumstances that may jeopardize the confidentiality of information, offered by patients to their health providers. Therefore, some effects of the current Chilean law are analyzed in this respect, regarding access to data from the clinical record of a patient. Also, the risks of putting certain data on social networking sites are analyzed, as well as some of its effects on clinical practice. The reasons because of mandatory reporting of diseases, meaning danger to public health, is allowed, are mentioned. We also discuss the difficulties involved in managing the results of preventative health screenings and its knowledge by third parties, as well as some possible violations of personal privacy, regarding dissemination of some people health information and its further mention or figuration in mass media. We conclude that it is a must for both physicians and other health team members, to safeguard confidentiality of data to which they have had access, as well as the need to know the relevant law, in order to respect human dignity of patients, each one as a person. We address the attention to the possibility that, practicing in a different way, it could endanger the reliability of clinical records, also impairing the quality of people's health care.

(Rev Med Chile 2015; 143: 358-366)

Key words: Access to Information; Confidentiality; Information Dissemination; Medical Records; Social Networking.
$\mathrm{E}$ n una comunicación previa, hemos revisado la vigencia y necesidad de la confidencialidad del acto médico ${ }^{1}$, expresión del respeto a la intimidad del paciente. A partir de lo allí concluido examinaremos en este trabajo algunas situaciones específicas que, hoy en día, colocan bajo apremio o dificultan la mantención del debido secreto, en relación con las acciones que competen a profesionales de la salud y sus pacientes.

Las actuales condiciones de vida, la amplia disponibilidad de nuevas tecnologías, la necesidad de atender muchas personas en las instalaciones sanitarias, la necesaria concurrencia de diversos especialistas, las necesidades propias de la ense- ñanza de la medicina, una opinión pública que valora la transparencia así como la presencia y acción de los medios de comunicación masiva, entre otros puntos, generan circunstancias que ponen en riesgo la debida reserva en la mantención de datos e información de carácter confidencial. Las obvias repercusiones de lo anterior en una adecuada relación médico-paciente, hacen necesario reflexionar sobre estos temas para considerar la forma de manejarlos, teniendo presentes la dignidad y el bienestar de los pacientes, sin descuidar las buenas prácticas en su atención.

Con el propósito de contextualizar adecuadamente el problema, creemos necesario iniciar el 
presente análisis con un comentario sobre aspectos pertinentes de la ficha clínica.

Las primeras historias clínicas fueron descritas en el Corpus Hippocraticum, en Epidemias I y III, donde se recogen, ordenada y sistemáticamente, la caracterización personal y el modo de enfermar de cada paciente ${ }^{2}$. Sydenham, en sus Observationes Medicae (1676), propone que la descripción de las enfermedades se haga "tan gráfica y natural como sea posible", ordenando los casos de la experiencia clínica en especies, al modo de los botánicos ${ }^{3}$.

Laín Entralgo ${ }^{4}$ definió la historia clínica como el documento que recoge la información confiada por el enfermo al médico, para obtener el diagnóstico, tratamiento y posible curación de su enfermedad, guardando los hechos esenciales que configuran la situación particular.

Una buena historia clínica, tal como lo señalan Goic y Chamorro ${ }^{5}$, debe contener solamente datos confiables, no omitir ninguna información útil, ser concisa, libre de datos superfluos y objetiva. Incluirá el análisis y juicios clínicos que el propio médico introduce en el proceso ${ }^{6}$.

La atención individual de personas por parte de un médico origina la necesidad que éste tome nota atingente del relato del paciente, los hallazgos del examen físico, eventuales diagnósticos, tratamientos aplicados, evolución en el tiempo y pronóstico que se haya efectuado en relación con el caso específico. Esto se origina por razones de fidelidad con lo acaecido, número de consultantes, y el perfil temporal de la relación médico-paciente, lo que implica que el registro no esté solamente confiado a la memoria sino a un documento o documentos, que pueden ser consultados acorde con las necesidades de la atención médica. En la actualidad, se suma a estos argumentos el hecho que los pacientes pueden ser atendidos por múltiples profesionales, de diversas especialidades y dentro del contexto de instituciones prestadoras de salud, lo que hace imprescindible que se registre y guarde los datos e información generados en los sucesivos contactos de la persona con el sistema de salud.

Fue en la Clínica Mayo, Rochester, cuando en 1907 el Dr. Henry Plummer ${ }^{7}$ y su asistente Mabel Root inauguraron un sistema centralizado para la admisión y registro de los hechos clínicos de los pacientes, que luego se transformó en estándar mundial.

Surgió entonces un documento o expediente que contiene datos e información concernientes a una persona que ha efectuado una consulta al médico o sistema de salud. Éstos, por su propia naturaleza, constituyen información que se inscribe en la esfera de la privacidad personal, y es calificada hoy como sensible.

Pero, en esencia, ¿cuál es la naturaleza de una ficha clínica? Al respecto, nos parece necesario considerar que en su comienzo la historia clínica descansa en un componente oral donde, en el encuentro clínico, el paciente relata al médico el motivo de su consulta, a la vez que éste repregunta y explora alternativas relacionadas tanto con el relato actual como con los antecedentes del paciente, además de otros hechos individuales y del contexto socio-económico, los que ayudan a configurar la totalidad del consultante en su condición de persona.

A lo anterior, se agrega el registro de un cuidadoso examen físico, general y segmentario, luego que el médico lo ha efectuado. Posteriormente se añaden una o varias hipótesis diagnósticas y un plan de trabajo, que comprende lo terapéutico y lo exploratorio en términos de procedimientos y diversos exámenes de laboratorio.

Para guardar fidelidad con lo acaecido con un paciente en particular, y contribuir a la calidad y seguridad de la atención, resulta imprescindible dejar un registro escrito de los distintos momentos de la enfermedad y/o consulta. Por tanto, la historia clínica se configura como una suerte de memoria auxiliar del médico tratante.

Por otra parte, en la historia clínica se colocan datos que deben ser compilados y analizados de un modo global, reflexionando sobre la calidad de los mismos, su pertinencia y la forma como se interrelacionan, de modo de lograr una conclusión, que puede tener valor temporal o definitivo, según el estado de evolución de la enfermedad particular de ese paciente en concreto. Ello permitirá llegar a un diagnóstico y planificar una terapéutica concordante con los hechos. Por tanto, la historia clínica es un instrumento fundamental de reflexión y trabajo médico, con connotación asistencial objetiva.

La vida humana tiene dimensión temporal, al igual que las enfermedades. En la historia clínica, de allí esa denominación, va quedando el relato cronológico de lo acaecido. Ello permitirá objetivar la evolución del paciente e intentar comprender lo que sucede, en escala temporal. De ese modo la historia clínica permite dilucidar, en el 
tiempo, lo mejor que se le puede ofrecer al paciente individual, tamizando información del medio, su genética personal y eventuales intervenciones terapéuticas. Es decir, se trata de algo claramente orientado al registro de los actos médicos que han tenido lugar durante la vida de una persona.

Cuando el paciente necesita la asistencia de diferentes médicos, sus antecedentes clínicos, reunidos en un sitio común, serán de utilidad para tomar conocimiento adecuado y oportuno de los hechos y condiciones que configuran la enfermedad de esa persona en un momento dado de su vida, sea en la práctica hospitalaria o en la ambulatoria. Adicionalmente, allí quedarán registrados los hechos que tengan que ver con cada una de las acciones efectuadas por los diferentes especialistas que han atendido al enfermo, como también los controles de constantes vitales, así como el cumplimiento de las indicaciones médicas, permitiendo que el médico tratante pueda quedar informado de lo efectuado. Podemos concluir que, una buena historia clínica es un conjunto de documentos y registros que permiten la adecuada integración y coordinación médica, lo que irá en directo beneficio para el paciente.

¿Cuál es, entonces, el objetivo principal de la ficha clínica? Ciertamente, su orientación está dirigida a posibilitar el mejor manejo de los problemas de salud de un paciente que consulta, recogiendo y proporcionando información veraz, oportuna y agregada, para obrar acorde con las reglas del arte médico y las factibilidades existentes. Secundariamente, puede permitir obtener datos epidemiológicos, contribuir a la docencia e investigación clínica, evaluar resultados de tratamiento y calidad de la atención, servir de referencia para situaciones médico-legales y/o permitir la obtención de información científica.

Si la ficha clínica se orientara al mero registro de datos, con o sin interpretaciones de resultados de análisis, bajo una mirada orientada con mayor énfasis a la ley en vez de la clínica médica, tendería a ser construida con la atención puesta en las eventuales consecuencias de lo registrado en vez de favorecer el proceso diagnóstico y/o terapéutico. Así, podría haber integrantes del equipo médico que se abstuvieran de opinar o que no quisieran dar datos relacionados con la condición particular de un enfermo, para no verse involucrados judicialmente. En otro plano, pudiera ser que no se hicieran todas las discusiones o interpretaciones transitorias adecuadas, con los elementos que están disponibles en ese momento, para evitar malas interpretaciones a posteriori, sea por legos o letrados. Adicionalmente, habría datos no registrados, por constituir -por ejemplo- un menoscabo para la honra o prestigio del paciente.

Puede concluirse, entonces, que esta modalidad judicializada no resulta conveniente para los intereses del paciente ni del tratante, lesionando gravemente el acto médico.

En Chile, la ficha clínica está definida legalmente en el Artículo $12^{\circ}$ de la ley $20.584^{8}$. Esos documentos, donde se registren procedimientos $y$ tratamientos a los que fueron sometidas las personas, serán considerados como dato sensible, de conformidad con lo dispuesto en la letra g del artículo 2º de la ley No 19.628, de 1992.

En otro plano, al considerar la ficha clínica, por una parte como un instrumento elaborado acorde con datos y observaciones recogidas por los profesionales de la salud en la forma más fidedigna, y por otra, con la finalidad de mejorar la atención del enfermo, se constituye en apoyo imprescindible al trabajo clínico. Inevitablemente, contendrá numerosos datos necesarios de ser protegidos por su naturaleza confidencial ${ }^{9} \mathrm{y}$, en términos bioéticos, está básicamente orientada y regida por el principio de la beneficencia. Por ello se considera, desde el punto de vista médico, tal como lo señala Vacarezza ${ }^{10}$, un "instrumento privado", propiedad del profesional o institución que cobija la atención.

Dado que la ficha clínica cumple también con una finalidad en el área docente y de investigación, el acceso a ella debe estar permitido con esos fines, pero con las limitaciones que cautelen la seguridad y privacidad de su contenido.

El antiguo principio hipocrático de guardar el secreto, en lo que al paciente se refiere, ha sido recogido en códigos de ética médica de diversos países, pudiendo señalarse a modo de ejemplo y sin pretender ser exhaustivos, los casos de Chile ${ }^{11}$ y Argentina ${ }^{12}$, donde se explicita que es obligatorio incluso hasta después de la muerte del enfermo. En España ${ }^{13}$ también se muestra la confidencialidad como exigible, misma situación que se señala en los Estados Unidos de América ${ }^{14}$ y en Canadá ${ }^{15}$. La Asociación Médica Mundial, en la Declaración de Ginebra, exige "guardar y respetar los secretos confiados a mí, incluso después del fallecimiento del paciente"16, desde su versión inicial en 1948, 
manteniendo el concepto en sucesivas revisiones. Análogas declaraciones se hicieron para los datos de investigación y son considerados dentro de los derechos de los pacientes ${ }^{17,18}$.

Tener acceso a la ficha clínica lleva implícito el compromiso moral, de quien lo hace, además del establecido en las leyes, de guardar completa reserva de su contenido, protegiendo tanto a los pacientes como a terceros que aporten datos. Ello alcanza, obviamente, a cualquier comentario que se pueda verter sobre un paciente en un medio no clínico.

\section{La ley 20.584}

Esta ley permite, desde 2012, amplio acceso del paciente a la ficha clínica y la posibilidad de requerir copia de la misma. Esta disposición, conocida coloquialmente como "Ley de Derechos y Deberes del Paciente", establece en su artículo 13 que la información registrada en la ficha, "copia de la misma o parte de ella, será entregada, total o parcialmente" a una serie de personas en caso de "solicitud expresa"19. Entre estas personas se cuentan el paciente, los Tribunales de Justicia (que pueden requerir la información guardada en ella, al tenor de los dispuesto en el art. 13, letra c) o instituciones relacionadas. $\mathrm{Al}$ respecto, desde el punto de vista ético, cabe mencionar que esto es lícito cuando se trata de un requerimiento de tribunal competente, en virtud de ley legítima y que la información que se entregue debe ser únicamente la necesaria de conocer, para resolver en justicia el requerimiento. En Chile, se menciona explícitamente que esto debe darse solamente cuando la información contenida en la ficha clínica se relacione con las causas que se estuvieren conociendo; para el caso de los fiscales y abogados ello será "cuando la investigación se vincule directamente con las investigaciones o defensas que tengan a su cargo" 20 .

En una especificación significativa se agrega que también se entregará a sus herederos, en caso de fallecimiento del paciente. Parece aquí haber primado un sentido litigante, sobre posibles o eventuales acciones médicas sujetas a crítica, más que la conveniencia del paciente. Sin embargo, cabe considerar que el término "herederos" abarca un espectro muy amplio, que va desde los hijos, cónyuge, terceros relacionados en diverso grado, pudiendo llegar hasta el Fisco, en caso de herencias intestadas. Esto puede poner en manos de terceros información que el titular de la misma entregó de modo completamente reservado a su(s) médico(s) tratante(s) y que, eventualmente, puede afectar su imagen $\mathrm{u}$ honra, como fueron conocidas durante su vida. En este sentido, señalamos a modo de ejemplo, que la ley considera, en Puerto Rico ${ }^{21}$, el 'derecho a la imagen', y en el caso de España ${ }^{22}$, los derechos al honor, la intimidad y la propia imagen están señalados en la Constitución de ese país.

Pero, dado que el secreto médico no incluye a la familia del paciente mientras él vive, esto implica, en los hechos, la terminación del secreto con la muerte del enfermo. Ello, sin embargo, entra en contradicción frontal con el sentido común, que considera que el respeto debido a la honra de una persona se extiende más allá de su vida; con la norma invariable de la tradición del ejercicio de la medicina; con los Códigos de Ética profesionales nacionales ${ }^{23}$ e internacionales ${ }^{24-26}$, tanto de organizaciones médicas como de otras profesiones relacionadas a la salud ${ }^{27}$ y de otras profesiones que toman contacto con información considerada como privada ${ }^{28}$; $y$, en general, con el consenso académico en relación al tema ${ }^{29-33}$. Esta norma se encuentra también en cierta tensión con la definición de la honra y el respeto a la vida privada como derechos constitucionalmente consagrados $^{34}$.

Una posible solución de tipo legal al problema, pasa por propugnar una interpretación restrictiva de esta norma, la que debe ser estudiada por los expertos correspondientes. En efecto, debe tenerse en cuenta que los secretos de la persona contenidos en la ficha no son propiamente parte de su patrimonio y, por lo tanto, no son sujetos de herencia. Por lo tanto, no puede decirse que los deudos de un paciente sean dueños, en sentido propio, de los datos de la ficha clínica. Podrían recibir sólo parte de la ficha. En este caso, aquella parte de la que pueda darse alguna de las siguientes condiciones: (i) consentimiento presunto del paciente para divulgar los datos; (ii) protección de la salud de terceros, como en el caso de posibles enfermedades hereditarias; (iii) pertinencia para decidir asuntos hereditarios y (iv) los límites comunes a la confidencialidad de la ficha relacionados con la administración de justicia* ${ }^{*}$.

Se puede aducir que, muerta la persona desaparecen sus derechos, ya que al no existir ésta 
no hay sujeto de derechos. Sin embargo, esta observación no exime al equipo de salud, a las personas en formación y, particularmente, al médico, de la promesa de confidencialidad hecha a su paciente, de cuya obligación sólo podría ser liberado por este último, lo cual es materialmente imposible. Cabe considerar la pertinencia, o no, de que cada persona pueda pronunciarse mientras sea competente, dejando instrucciones específicas en el sentido de qué hacer con su ficha clínica al fallecer, de manera que establezca si los datos en ella contenidos pueden, o no, ser de conocimiento de sus herederos o quienes tengan acceso a la información, cautelando la honra y memoria a la que toda persona aspira. Ello no quita validez alguna a la obligación primaria del médico de guardar la confidencialidad.

\section{Las redes sociales}

El mundo virtual de relaciones interpersonales que se ha construido gracias a las redes sociales basadas en la "World Wide Web", vía Internet, ha alcanzado vastos ámbitos de la vida comunitaria en las sociedades occidentales contemporáneas, en un grado de profundidad superlativo. En efecto, no sólo se trata ya de la cantidad de personas inscritas en estas redes, sino de la variedad e intimidad de los datos que comparten. Facebook tiene, por ejemplo, más de 1.000 millones de usuarios activos alrededor del mundo ${ }^{35}$, mientras que en Chile se calcula que entre $40 \%$ y $60 \%$ de la población está inscrita en este $\operatorname{sitio}^{36,37}$ y hasta $80 \%$ de los jóvenes entre 18 y 29 años $^{38}$.

Los médicos y otros profesionales del equipo de salud no están exentos de esta tendencia. Por una parte estos medios de comunicación virtuales ofrecen valiosas oportunidades de contacto con los pacientes que permiten mantener interacciones educativas y terapéuticas más estrechas en una amplia variedad de campos de la medicina ${ }^{39-41}$. Sin embargo, la participación de los profesionales de la salud en las comunicaciones en línea plantea también dificultades especiales de las que ellos no siempre están conscientes. El alcance y la perdurabilidad de lo difundido a través de Internet es mucho mayor a lo que estamos habituados. Hasta algunos años la mayor preocupación de un médico en este sentido podía ser que alguien escuchara al pasar algún comentario reservado hecho liviana- mente en un sitio público. Ahora, todo lo escrito en Facebook o Twitter puede alcanzar en minutos a millones de personas. Además, su registro puede ser recuperado en cualquier momento futuro. Sin embargo, la conducta de los usuarios de estos medios no refleja preocupación por este hecho. Erróneamente se puede tener la falsa e injustificada sensación de no estar expuesto públicamente, lo que tiende a propiciar una conducta más desinhibida que en la comunicación tradicional cara a cara ${ }^{42,43}$.

El impacto de estas tendencias comunicacionales en la preservación de la privacidad del paciente no ha sido detalladamente cuantificado aún, sin embargo, hay reportes, cada vez más frecuentes, de conductas poco profesionales en estos ámbitos. En Estados Unidos, donde el tema ha sido más estudiado, se han descrito casos de estudiantes y médicos que han sido sancionados, o se han expuesto a ello, por divulgar información médica de pacientes en YouTube o Facebook con un nivel de detalle suficiente para permitir su identificación por otras personas ${ }^{44,45}$.

A pesar de ello, la prevalencia general de conductas poco profesionales en comunidades virtuales no cesa de crecer $^{46}$. En este sentido, una serie de comportamientos que pueden parecer inocentes, pero que faltan a la prudencia, pueden conducir a múltiples problemas, tales como establecer inapropiados lazos de amistad con un paciente, estimar que ya no hay obligación de guardar el secreto profesional al existir informaciones sobre ese enfermo en la red, emitir comentarios sobre la situación que se vive en una organización o particular situación asistencial, tomar conocimiento de detalles de la vida de la persona no confiados por ésta y ajenos a la relación médico-paciente, vulnerando así la necesaria confianza entre el profesional y quien consulta.

Dadas las características de la comunicación digital, la observación cuidadosa de una conducta ética profesional tiene en este caso una naturaleza e importancia especial. La ubicuidad de los dispositivos que permiten difundir y leer contenido explícito en línea; la sensación injustificada y equívoca de privacidad e intrascendencia de las conductas asumidas en estos sitios; $y$, por otra parte, la persistencia temporal y el registro universal de lo publicado, son situaciones que configuran un nuevo escenario para el ejercicio de la medicina de nuestros días, en el cual, como en ningún otro, se 
demuestra con claridad meridiana la necesidad del compromiso por respetar la dignidad del paciente y su entorno familiar en todo momento. En otras palabras, surge un nuevo desafío en relación a la comprensión del secreto médico, la intimidad del paciente y el respeto por su autonomía.

Haciendo eco de recomendaciones internacionales en este tema ${ }^{47}$ se pueden considerar, entre otras, algunas precauciones como estar permanentemente consciente de la magnitud y alcance de las redes sociales, como de las potenciales fallas a la seguridad en las mismas, evitando conductas equívocas que permitan alterar los límites naturales de la relación médico-paciente, protegiendo así la dignidad de las personas, la confianza pública en la profesión médica y la eficacia de las acciones profesionales.

\section{Las enfermedades de notificación obligatoria}

En Chile el Decreto Supremo $\mathrm{n}^{\circ} 158$, del 22 de octubre de 2004, establece las patologías, modalidad y responsables de notificar a la Autoridad Sanitaria de ciertas enfermedades, consideradas peligrosas para la salud pública.

Desde el punto de vista ético, hemos señalado previamente ${ }^{1}$ que esta situación debe considerarse desde el bien común. Lo anterior implica, necesariamente, tomar los resguardos para defender la dignidad de las personas involucradas, entregando sólo la información indispensable para atender debidamente a la situación.

Cabe considerar también lo señalado por Altisent ${ }^{48}$ que la lealtad del médico hacia sus pacientes tiene fundamento en el respeto para la dignidad de aquellos y que esa lealtad profesional, para ser auténtica, está orientada al bien, por lo que no puede ser exigida para cooperar a la injusticia.

\section{Los seguros y exámenes preventivos}

Otro problema digno de considerar es el relacionado con las autorizaciones que entregan, obligados por contratos de adhesión, los pacientes y/o potenciales enfermos a compañías de seguros, con el fin de permitir el acceso a sus datos clínicos frente al reclamo de un siniestro, como se denomina el evento que origina la petición del contratante para concurrir económicamente a solventar los gastos derivados de una enfermedad o accidente. No cabe duda que es lícito que se solicite prueba de que lo pedido corresponde a lo contratado, pero debe reconocerse que la autorización a que se obliga al contratante es de mucha mayor extensión, existiendo dudas fundadas sobre los grados de libertad con que se accede al pedido de la aseguradora. Adicionalmente, información confidencial puede llegar a manos de personas que, ejecutando diversas labores, no crean, sepan o estén dispuestas a guardar reserva sobre ella, con grave riesgo para los afectados por esa conducta.

Las personas sometidas a exámenes preventivos, o de aptitud para un determinado trabajo, lo hacen en el contexto de averiguar si presentan o no enfermedad que sea un riesgo para su propia salud o la de terceros. Es por ello que deben autorizar que esos resultados sean conocidos por terceros. Ello no es un problema cuando se trata solamente de un médico, que le podrá recomendar acciones terapéuticas o preventivas para el cuidado de su propia salud, pero sí lo es -potencialmente- cuando se trata de personas ajenas a la medicina, como es el caso del actual o potencial contratante de los servicios, incluyendo la posibilidad de quedar sin trabajo. Sin embargo, es necesario considerar en este caso una condición de beneficencia y no maleficencia, cuando el trabajo pueda provocar un daño serio a la salud de esa persona, agravando una condición preexistente o generando una enfermedad no presente, o dañando la salud de otros en el trabajo, por falta de habilidades psico-físicas para ejercerlo. También se debe considerar el resultado de los exámenes y tratamientos ocurridos a raíz de enfermedades profesionales o accidentes del trabajo, donde es indispensable tener una información apropiada para poder definir la vuelta al trabajo de la persona involucrada o una posible reubicación o cambio de faena. Lo anterior implica, ciertamente, el uso de esa información con gran discreción, de manera que el conocimiento se limite severamente a quienes estrictamente deban saber y se mantenga cautelada la dignidad personal del involucrado.

\section{El control de calidad y rendimiento clínico}

Se ha utilizado el acceso a la historia clínica con el objeto de verificar el cumplimiento de normas, estándares de atención $\mathrm{u}$ otros, tanto para fines relacionados con la calidad de la atención que re- 
ciben los pacientes, como para recoger datos sobre rendimiento de los profesionales involucrados en la atención de aquellos.

Si se audita con el objeto de verificar que la atención se haga tal y como está definido por la norma, cuando la haya, se puede dar un efecto beneficioso, tal como permitir que los pacientes reciban tratamiento acorde con las evidencias existentes. También, y esto se acerca más a la investigación sobre servicios de salud, se puede verificar la efectividad, o ausencia de ella, de los tratamientos prescritos. En todos estos casos se debe mantener la confidencialidad de lo conocido y evitar que se den a conocer datos de pacientes que no han dado su autorización para ello.

Un sano control, tendiente a cautelar el debido uso y rendimiento de los recursos, es necesario tanto en salud como en numerosas otras actividades. Sin embargo, cuando el propósito del análisis es meramente establecer rendimientos cuantitativos, olvidando que la medicina es significativamente arte, se desvía de la finalidad a la cual debiera servir.

Si se quiere tender a 'optimizar' numéricamente las atenciones, si se privilegia la cantidad sobre la calidad, o solamente se quiere establecer el número y tipo de recursos como exámenes u otros utilizados, únicamente para estimar cuanto 'produce' cada miembro del equipo de salud, sin considerar las peculiaridades de cada caso, se puede irrumpir indebidamente en la confidencialidad del acto médico. Con ello no se considera el bien común ni al enfermo, sino solamente las conveniencias de diversos grupos de influencia, a veces, con motivaciones ajenas al bienestar de las personas que recurren a la atención médica.

\section{Los accidentes y hechos de conocimiento público}

Debe plantearse también lo que sucede en ciertas ocasiones que suscitan el interés del público, como es el caso de accidentes que generan uno o múltiples lesionados y, debido a su espectacularidad, impacto o compromiso de la seguridad pública, entre otras razones, promueven el interés de los medios de comunicación masiva, interesándose por las imágenes o informaciones que dicen relación con las lesiones sufridas por las víctimas y su pronóstico. Similares acciones se aprecian también cuando los involucrados tienen, por cualquier causa, la condición de 'figura pública' o cuando se trata de generar opinión frente a determinadas circunstancias, por ejemplo, respecto de la necesidad de órganos para trasplantes. De igual forma, cuando se trata de casos considerados 'raros', sea por lo inhabituales o por las consecuencias de la enfermedad. Es de público conocimiento la frecuencia con que se informa con toda clase de detalles, incluyendo 'partes médicos' generados por las autoridades de las organizaciones que actúan como prestadoras, a veces exigidos en nombre de la 'transparencia', sin considerar mayormente los sentimientos y deseos de la familia, o del propio afectado, si éste estuviere en condiciones de expresarlos. Pareciera, en ocasiones, que la curiosidad pública es mayor que la obligación de mantener la privacidad de las personas y proteger su intimidad. Estimamos que, en estos casos, debiera contarse previamente, con la autorización explícita del afectado o su representante legal, recogiendo la confidencialidad como un derecho del paciente, como lo señalara Vacarezza ${ }^{49}$.

A este respecto, también cabe considerar el hecho que es posible el uso sesgado de la información, con el propósito de causar alarma pública y/o generar simpatías (o antipatías) frente a una determinada situación o, simplemente, "crear" una noticia sin que sea posible, para quienes no tienen acceso a la información completa, comprender la verdad de los hechos. Lo anterior, puede constituir manipulación de la comunidad que desea conocer un tema, y es, a nuestro juicio, moralmente inapropiado puesto que el objeto de la noticia no es sólo informar, sino eventualmente, ser concurrente con los intereses del informante.

\section{Conclusión}

A pesar que la confidencialidad de los datos relacionados con el acto médico debe ser mantenida, por respeto a la dignidad de la persona que ha otorgado acceso a su intimidad, nos encontramos a diario con situaciones donde se exige acceso a los mismos, con variados pretextos.

Constituye un deber relevante para los médicos y otros miembros de los equipos de salud cuidar lo confiado y tomar las medidas necesarias para ello, junto con conocer la legislación pertinente, en la medida que corresponde a un ciudadano. 
La autorización previa para revelar información sensible de los pacientes es indispensable, salvo en las circunstancias que se han exceptuado previamente, como es el caso de aquellas situaciones que ponen en peligro la sanidad pública. Adicionalmente, se estima necesario estudiar algunas modificaciones legales que garanticen, a los involucrados, la mantención de su imagen y honor una vez fallecidos, regulando el acceso a datos e información clínica recogida mientras estaban con vida.

En la obtención, registro y utilización de la historia clínica se debe cautelar apropiadamente la confidencialidad por respeto a la persona del paciente. Secundariamente, si ello no es efectivo, se tenderá a disminuir la cantidad y confiabilidad de los datos que contiene, en particular aquellos provenientes de terceros o que por su naturaleza sea delicado se den a conocer a personas no autorizadas, atentando contra su estructura y contenidos esenciales, disminuyendo significativamente la calidad de la atención que recibirá un enfermo.

${ }^{*}$ Agradecimientos: Agradecemos a los profesores Cristián Larraín Páez, de la Facultad de Derecho de la Universidad del Desarrollo, y Antonio Bascuñán Rodríguez, de la Facultad de Derecho de la Universidad Adolfo Ibáñez por su orientación en este punto. Lo consignado en el texto, sin embargo, es de exclusiva responsabilidad de los autores.

\section{Referencias}

1. Grupo de Estudios de Ética Clínica de la Sociedad Médica de Santiago. El respeto a la intimidad del paciente. Rev Med Chile 2014; 142: 506-11.

2. Fombella MJ, Cereijo MJ. Historia de la historia clínica. Galicia Clin 2012; 73 (1): 21-6.

3. Citado en 2.

4. Lain Entralgo P. La Historia Clínica. Ed. Triacastela, Madrid, 1998.

5. Goic A, Chamorro G. Semiología Médica. Publicaciones Técnicas Mediterráneo, Santiago de Chile. 1987. Cap. 19.

6. Pérez I. La Historia Clínica. Tesis presentada para obtener el título de Magíster en Bioética. Pontificia Universidad Católica de Chile, Facultad de Medicina. 2008.

7. Mayo Clinic. Tradition and Heritage. Disponible en: http://www.mayoclinic.org/tradition-heritage/medicalrecords.html (Consultado el 08 de abril de 2013).

8. República de Chile. Ministerio de Salud. 24 de abril de
2012. Ley núm. 20.584. Regula los derechos y deberes que tienen las personas en relación con atenciones vinculadas a su atención en salud.

9. Vega SJ. ¿A quién pertenece la ficha clínica? Rev Med Chile 2003; 131: 1469-70.

10. Vacarezza R, Núñez E. ¿A quién pertenece la ficha clínica? Rev Med Chile 2003; 131: 111-4.

11. Colegio Médico de Chile. Código de Ética. En: http:// www.colegiomedico.cl/Portals/0/files/etica/120111 codigo_de_etica.pdf (Consultado el 05 de octubre de 2013).

12. Confederación Médica de la República Argentina. Código de Ética. En: http://colmedse.com.ar/docs/codigoetica.pdf (Consultado el 05 de octubre de 2013).

13. Consejo General de Colegios Oficiales de Médicos. Código de Deontología Médica. Madrid, España. Julio de 2011. En: https://www.cgcom.es/sites/default/files/ codigo_deontologia_medica.pdf (Consultado el 05 de octubre de 2013).

14. American Medical Association. AMA's Code of Medical Ethics. En: http://www.ama-assn.org/ama/pub/ physician-resources/medical-ethics/code-medical-ethics/ opinion505.page? (Consultado el 5 de octubre de 2013).

15. Kleinman I, Baylis F, Rodgers S, Singer P. Bioethics for clinicians. 8. Confidentiality. Can Med Assoc J 1997; 156 (4): 521-4.

16. Asociación Médica Mundial. Declaración de Ginebra. En: http://www.unav.es/cdb/ammginebral.html (Consultado el 05 de octubre de 2013).

17. Asociación Médica Mundial. Declaración de Helsinki. Disponible en: http://www.wma.net/es/30 publications/10policies/b3/ (Consultado el 02 de noviembre de 2013).

18. Asociación Médica Mundial. Declaración de Lisboa. Disponible en: http://www.colegiomedico.cl/Default. aspx?tabid=236 (Consultado el 02 de noviembre de 2013).

19. Ibid. 8

20. Ibid. 8

21. Estado Libre Asociado de Puerto Rico. Ley número 139. Ley del Derecho sobre la Propia Imagen del 13 de julio de 2011. En: http://www.lexjuris.com/lexlex/Leyes2011/ lexl2011139.htm. (Consultado el 05 de octubre de 2011).

22. Constitución Española. Artículo 18. En: http://www. congreso.es/consti/constitucion/indice/sinopsis/sinopsis.jsp?art $=18 \&$ tipo $=2$ (Consultado el 05 de octubre de 2013).

23. Colegio Médico de Chile. Código de Ética. En: http:// www.colegiomedico.cl/Portals/0/files/etica/120111 codigo_de_etica.pdf (Consultado el 05 de octubre de 2013). 


\section{DOCUMENTOS}

Amenazas a la confidencialidad en medicina - Grupo de Estudios de Ética Clínica de la Sociedad Médica de Santiago

24. Consejo General de Colegios Oficiales de Médicos. Código de Deontología Médica. Madrid, España. Julio de 2011. En: https://www.cgcom.es/sites/default/files/ codigo_deontologia_medica.pdf (Consultado el 05 de octubre de 2013).

25. American Medical Association. AMA's Code of Medical Ethics. En: http://www.ama-assn.org/ama/pub/ physician-resources/medical-ethics/code-medical-ethics/ opinion505.page? (Consultado el 5 de octubre de 2013).

26. American College of Physicians Ethics Manual: sixth edition. Snyder L; American College of Physicians Ethics, Professionalism, and Human Rights Committee. Ann Intern Med 2012; 156 (1 Pt 2): 73-104.

27. Colegio de Psicólogos de Chile. Código de Ética. Disponible en http://www.colegiopsicologoschile.org/ (Consultado el 11 de abril de 2013).

28. Colegio de Abogados de Chile. Código de ética Profesional. Disponible en http://www.colegioabogados.cl/ cgi-bin/procesa.pl?plantilla $=/$ archivo.html\&bri $=$ cole gioabogados $\&$ tab $=$ art_1\&campo $=c \_$archivo $\& i d=747$ (Consultado el 11 de abril de 2014).

29. Ploem C. Medical confidentiality after a patient's death, with particular reference to The Netherlands. Med Law 2001; 20 (2): 215-20.

30. Werth JL, Burke C, Bardash RJ. Confidentiality in endof-life and after-death situations. Ethics Behav 2002; 12 (3): 205-22.

31. Bongers LM. Disclosure of medical data to relatives after the patient's death: recent legal developments with respect to relatives' entitlements in the Netherlands. Eur J Health Law 2011; 18 (3): 255-75.

32. Louvrier P. Professional confidentiality and the family. Rev Med Brux 2006; 27 (4): S396-400.

33. Bascuñán A. Deber de confidencialidad y secreto profesional del abogado. Revista de Estudios de la Justicia 2011 (15): 221-63. Disponible en http://web.derecho. uchile.cl/cej/rej15/BASCU\%C3\%91AN\%20_12_.pdf (Consultado el 11 de abril de 2014).

34. Constitución Política de la República de Chile, artículo 19\#4. Disponible en http://bcn.cl/1j3in (Consultado el 11 de abril de 2014).

35. “Facebook Reports First Quarter 2013 Results". Facebook. http://investor.fb.com/releasedetail.cfm?
ReleaseID=761090 Recuperado el 3 de julio de 2013.

36. http://en.wikipedia.org/wiki/Facebook_statistics

37. http://readwrite.com/2010/07/23/where_in_the_world_ is_facebook_used_interactive_ma\#awesm $=\sim$ obfI AhIxDBj65y

38. Encuesta Jóvenes y Participación Periodismo UDPFeedback 2011 http://www.prensafcl.udp.cl/encuestaperiodismo2011.pdf

39. Mellios A, Kolokathi A, Mechili A, Diomidous M. Use of social networks to inform the population about food borne disease. Stud Health Technol Inform 2013; 190: 258-60.

40. Shelby A, Ernst K. Story and science: How providers and parents can utilize storytelling to combat anti-vaccine misinformation. Hum Vaccin Immunother 2013; 9 (8): 1795-1801. doi:10.416/hv.24828

41. Goldstein K, Briggs M, Oleynik V, Cullen M, Jones J, Newman E, et al. Using digital media to promote kidney disease education. Adv Chronic Kidney Dis 2013; 20 (4): 364-9. doi: 10.1053/j.ackd.2013.04.001.

42. Suler J. The online disinhibition effect. Cyberpsychol Behav 2004; 7 (3): 321-6.

43. Aboujaoude E. Problematic Internet use: an overview. World Psychiatry 2010; 9 (2): 85-90.

44. New breed of doctors turn to social media, texting for patient care. June 11, 2012; www.cbsnews.com/2102504763_162-57450447.html?tag=contentMain;content body

45. Strausburg M. How Facebook almost ended my career with a single click. Acad Emerg Med 2011; 18: 1220.

46. Chretien KC, Gerysen SR, Chretien JP, Kind T. Online posting of unprofessional content by medical students. JAMA 2009; 302: 1309-15.

47. Shore R, Halsey J, Shah K, Crigger BJ, Douglas SP. AMA Council on Ethical and Judicial Affairs (CEJA). Report of the AMA Council on Ethical and Judicial Affairs: professionalism in the use of social media. J Clin Ethics 2011 Summer; 22 (2): 165-72.

48. Altisent R. Cuestiones éticas en el uso de la historia clínica. En: La implantación de los derechos de los pacientes. Pilar León Sanz, Ed. EUNSA, Navarra, 2004. pp. 307-25.

49. Vacarezza R. De los derechos del paciente. Rev Med Chile 2000; 128: 1380-4. 